EPJ Web of Conferences 13, 03001 (2011)

DOI: $10.1051 /$ epjconf/20111303001

(c) Owned by the authors, published by EDP Sciences, 2011

\title{
The Compressed Baryonic Matter Experiment at FAIR
}

\author{
Johann M. Heuser ${ }^{\text {a }}$ for the CBM Collaboration \\ GSI Helmholtz Centre for Heavy Ion Research GmbH, Darmstadt, Germany
}

\begin{abstract}
The Compressed Baryonic Matter (CBM) experiment is being planned at the international research centre FAIR, under realization next to the GSI laboratory in Darmstadt, Germany. Its physics programme addresses the QCD phase diagram in the region of highest net baryon densities. Of particular interest are the expected first order phase transition from partonic to hadronic matter, ending in a critical point, and modifications of hadron properties in the dense medium as a signal of chiral symmetry restoration. Laid out as a fixed-target experiment at the synchrotrons SIS-100/SIS-300, providing magnetic bending power of 100 and $300 \mathrm{~T} / \mathrm{m}$, the CBM detector will record both proton-nucleus and nucleus-nucleus collisions at beam energies up to $45 \mathrm{~A} \mathrm{GeV}$. Hadronic, leptonic and photonic observables have to be measured with large acceptance. The nuclear interaction rates will reach up to $10 \mathrm{MHz}$ to measure extremely rare probes like charm near threshold. Two versions of the experiment are being studied, optimized for either electron-hadron or muon identification, combined with silicon detector based charged-particle tracking and micro-vertex detection. The research programme will start at SIS-100 with ion beams between 2 and $11 \mathrm{~A} \mathrm{GeV}$, and protons up to energies of $29 \mathrm{GeV}$ using the HADES detector and an initial configuration of the CBM experiment. The CBM physics requires the development of novel detector systems, trigger and data acquisition concepts as well as innovative real-time reconstruction techniques. Progress with feasibility studies of the experiment and the development of its detector systems are discussed.
\end{abstract}

\section{The CBM physics programme}

The CBM experiment will conduct a comprehensive research programme on nucleus-nucleus collisions at FAIR [1-3]. The project aims at investigating the largely unexplored QCD phase diagram at highest net baryon densities and moderate temperatures (Fig. 1). The research is complementary to the heavy-ion programmes at RHIC and at LHC that address the physics of the early universe at low densities and high temperatures. The CBM experiment will be located at the SIS-300 synchrotron (Fig. 2). Projectile energies of $10-45 \mathrm{~A} \mathrm{GeV}$ will allow for creating in fixed-target collisions the highest net baryon densities, up to 10 times that of ground state nuclear matter. This will enable the CBM programme to focus on signatures of the expected first order phase transition from partonic to hadronic matter, ending in a critical point, and on modifications of hadron properties, e.g. their masses, in the dense nuclear medium as a signal of chiral symmetry restoration. The research programme will be started at the SIS-100 synchrotron, planned to be operational in the year 2018 [4]. The SIS-100 will deliver ion beams with energies between 2 and $11 A \mathrm{GeV}$, and protons up to $29 \mathrm{GeV}$ to the HADES detector [5] and an initial version of the CBM experiment. Recently proposed other programmes in this beam energy range at RHIC/BNL and NICA/JINR will be complementary to the CBM programme as they are limited in interaction rates and will focus on bulk particle production. The full exploration including rare probes will be the task of the CBM experiment at the future SIS-300 synchrotron.

\footnotetext{
a e-mail: J.Heuser@gsi.de
}

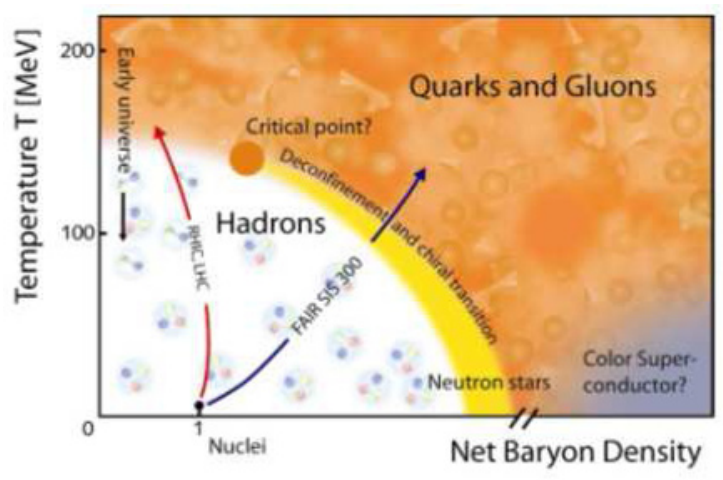

Fig. 1. The QCD phase diagram to be explored by CBM.

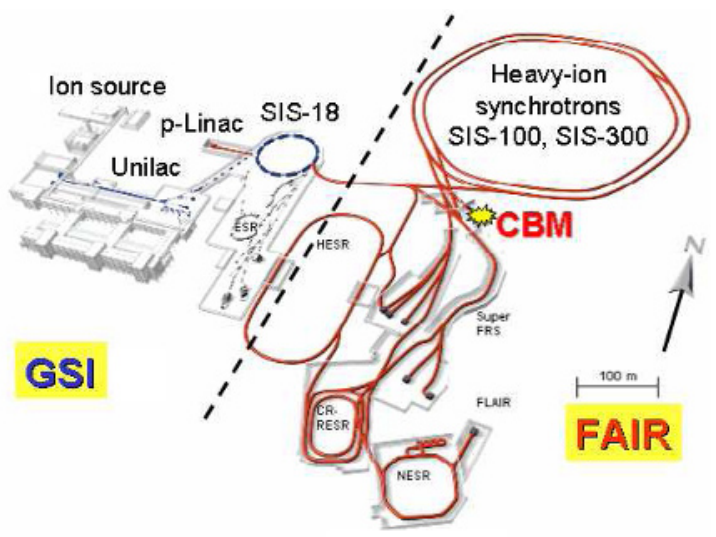

Fig. 2. The FAIR complex with the CBM experiment at the SIS-100 and SIS-300 synchrotrons.

This is an Open Access article distributed under the terms of the Creative Commons Attribution-Noncommercial License 3.0, which permits unrestricted use, distribution, and reproduction in any noncommercial medium, provided the original work is properly cited. 


\section{The CBM detector}

Two configurations of the CBM detector are being evaluated for electron-hadron and muon-hadron measurements. They are schematically shown in Fig. 3. Both may be realized at different stages. They have in common a lowmass silicon tracking system (STS), the central detector to perform charged-particle tracking and high-resolution momentum measurement with radiation tolerant silicon microstrip or pixel detectors. Combined with an ultra-thin micro-vertex detector (MVD) based on monolithic active pixel sensors, it will be installed in the gap of a dipole magnet in short distance downstream of the target, typically a gold foil of $250 \mu \mathrm{m}$ thickness corresponding to $1 \%$ nuclear interaction length. In the electron-hadron configuration, the CBM experiment comprises a ring imaging Cherenkov (RICH) detector downstream of the magnet to identify electron pairs from vector meson decays. Transition radiation detectors (TRDs) provide charged particle tracking and the identification of high energy electrons. Hadron identification will be realized in a time-of-flight (TOF) system built from resistive plate chambers (RPC). An electromagnetic calorimeter (ECAL) will be used for detecting direct photons. The projectile spectator detector (PSD) is a calorimeter that determines centrality and reaction plane of the collisions. In the muon-hadron configuration of the experiment, the RICH detector system is replaced by a compact active absorber system (MUCH). Vector mesons are detected via their decays into muon pairs. Hadrons can be measured with the absorbers moved out. A particular feature of the experiment is its data acquisition and trigger concept, imposed by the physics programme with rare probes, e.g. charm production near threshold, and the necessity for interaction rates between 0.1 and $10 \mathrm{MHz}$. It is based exclusively on self-triggering front-end electronics to time-stamp and to ship the detector signals to a fast computing farm for event building and high-level trigger generation.

\section{Physics performance studies and detector developments}

Progress with the preparation of the experiment has been achieved with detailed physics performance simulations, based on an increasingly realistic implementations of the CBM detector systems. This includes feed-back from the beginning detector developments and the evaluation of first demonstrator systems in test-beam experiments [6].

\subsection{Charged particle tracking}

The high multiplicity of up to 700 charged particles per central nuclear collision is reconstructed with the Silicon Tracking System [7]. The measurement of essentially all CBM observables depends on the high performance of the STS. Currently 8 tracking stations are considered in a $1 \mathrm{~T}$ dipole magnetic field based on radiation tolerant silicon micro-strip sensors mounted on a light-weight carbon fiber

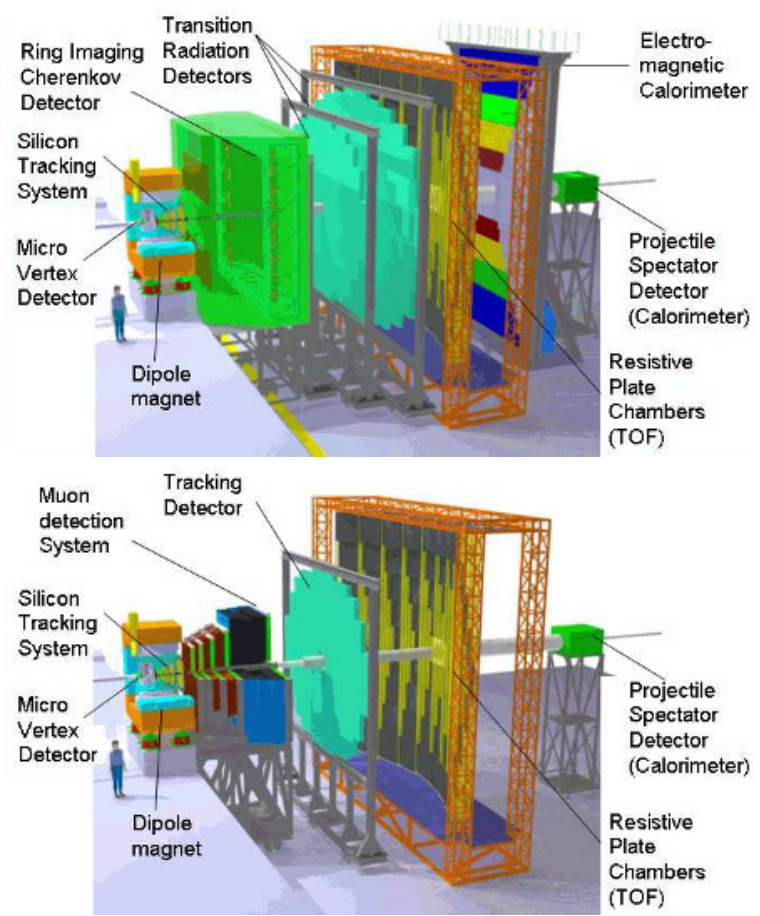

Fig. 3. The CBM detector in electron-hadron (top) and in muon configuration (bottom).

support structure. The material budget may be less then $1 \%$ radiation length per station. In central $25 \mathrm{~A} \mathrm{GeV} \mathrm{Au+Au}$ events, generated with UrQMD, tracks pointing to the primary vertex are reconstructed with $96 \%$ efficiency above momenta of $1 \mathrm{GeV} / \mathrm{c}$ using Cellular Automaton and Kalman Filter algorithms. The material budget is such that a momentum resolution of $1.3 \%$ is obtained.

\subsection{Di-lepton spectroscopy}

Electron identification is performed using a Ring Imaging Cherenkov Detector and a Transition Radiation Detector system. The $\mathrm{RICH}$ system uses a $\mathrm{CO}_{2}$ radiator of $1.5 \mathrm{~m}$ length, providing a pion separation threshold momentum of $4.65 \mathrm{GeV} / \mathrm{c}$. Mirrors of $12 \mathrm{~m}^{2}$ area project electron ring images of about $6 \mathrm{~cm}$ diameter onto the $2.4 \mathrm{~m}^{2}$ photo detection plane. The ring finding efficiency, evaluated in simulations with several reconstruction algorithms, is in excess of $95 \%$ for electrons embedded into central $\mathrm{Au}+\mathrm{Au}$ collisions at $25 \mathrm{~A} \mathrm{GeV}$ beam energy. The detector $\mathrm{R} \& \mathrm{D}$ for the RICH system focuses on the evaluation of thin spherical glass mirrors of $3 \mathrm{~m}$ radius with $\mathrm{Al}+\mathrm{MgF}_{2}$ coating and multi-anode photo multiplier tubes coupled to self-triggering readout electronics. For the TRD detector, innovative multi-wire proportional chambers with doublesided pad readout coupled to a foil radiator are being developed, capable of high counting rates. The combined electron identification efficiency of RICH and TRD is $85 \%$ at a pion suppression factor of $10^{4}$. The remaining background is dominated by $\pi^{0}$ Dalitz decays [8]. Muon identification is performed with a segmented hadron absorber and a 

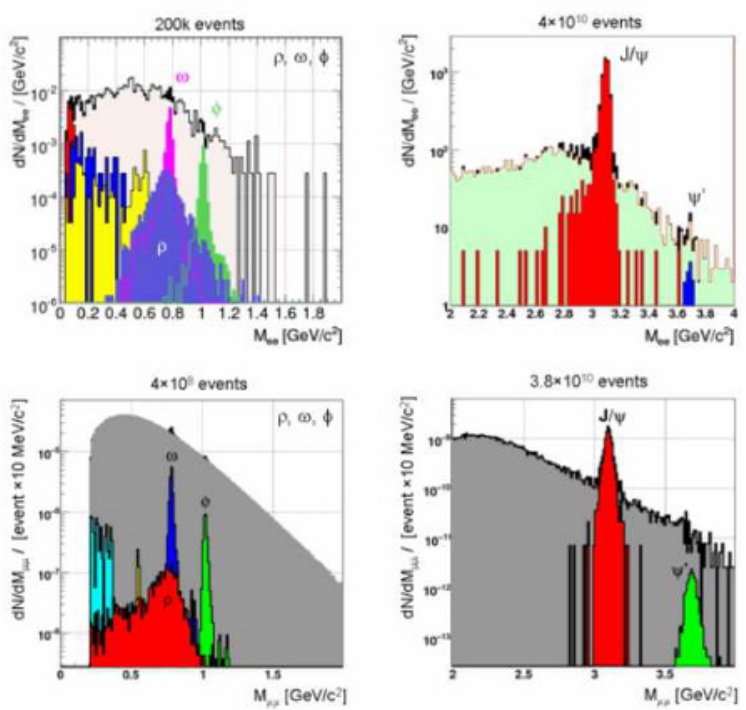

Fig. 4. Di-lepton spectra for low-mass vector mesons and charmonium, together with combinatorial background. The top two plots are for the di-electron experiment, the bottom two plots for the di-muon experiment.

tracking system downstream of the STS. The MUCH system is divided into two regions addressing low-mass muon pairs (vector mesons) and high-mass pairs $(\mathrm{J} / \psi)$. Five iron discs of $20 \mathrm{~cm}$ to $35 \mathrm{~cm}$ thickness, with a total thickness of 7.5 nuclear interaction lengths, are interleaved with 5 gaps of GEM detector layers and serve the measurement of the low-mass vector mesons. After another $1 \mathrm{~m}$ of iron and total 13.5 nuclear interaction lengths, the $\mathrm{J} / \psi$ pair candidates are detected. The particle multiplicity is reduced such that after $1.25 \mathrm{~m}$ iron 0.25 identified muons are obtained in $25 \mathrm{~A} \mathrm{GeV} \mathrm{Au}+\mathrm{Au}$ with the dominant background coming from $\pi$ and $\mathrm{K}$ decays at 0.13 muons per event [8]. Spectra and phase-space coverage of reconstructed di-leptons are shown in Figs. 4 and 5.

\subsection{Hadron measurement}

Hadrons are identified via time-of-flight measurement with a detector system based on resistive plate chambers. Different RPC technologies are being explored, including ultrathin glass pad RPC, ceramic RPC, and differential RPC based on semi-conductive glass. High counting rate capability up to $25 \mathrm{kHz}$ has to be achieved with a time resolution of 80 ps [9]. The "global" track reconstruction efficiency obtained with the simulated STS, TRD and TOF systems is $85 \%$. The phase-space coverage of reconstructed hadrons is shown in Fig. 6.

\subsection{Open charm detection}

The efficient separation of primary and short-lived decay vertices for open charm detection requires a $\mathrm{z}$-vertex resolution of the order of $50 \mu \mathrm{m}$ r.m.s. This is planned to be
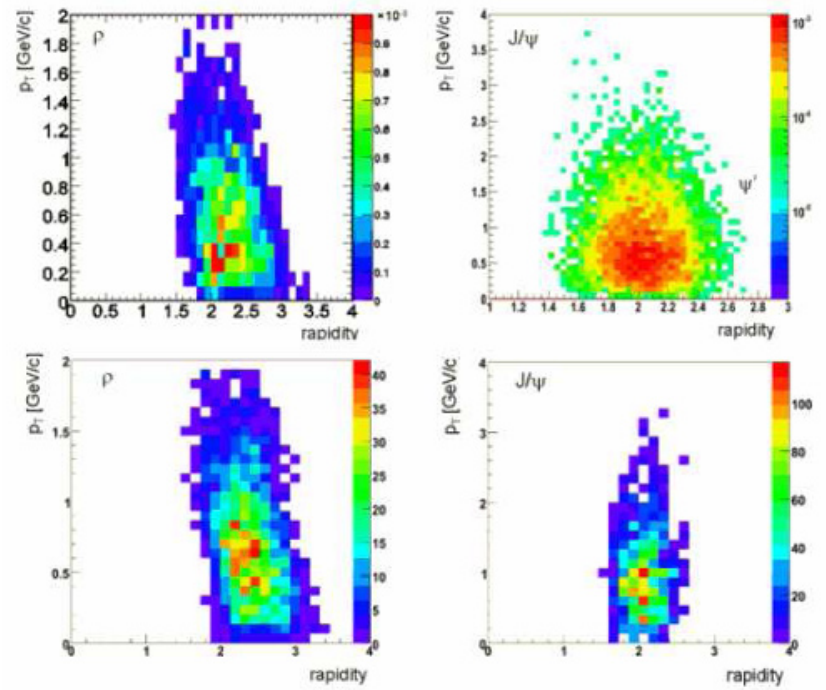

Fig. 5. Di-lepton phase space coverage for the electron configuration (top two plots) and the muon configuration of the CBM experiment (bottom two plots).

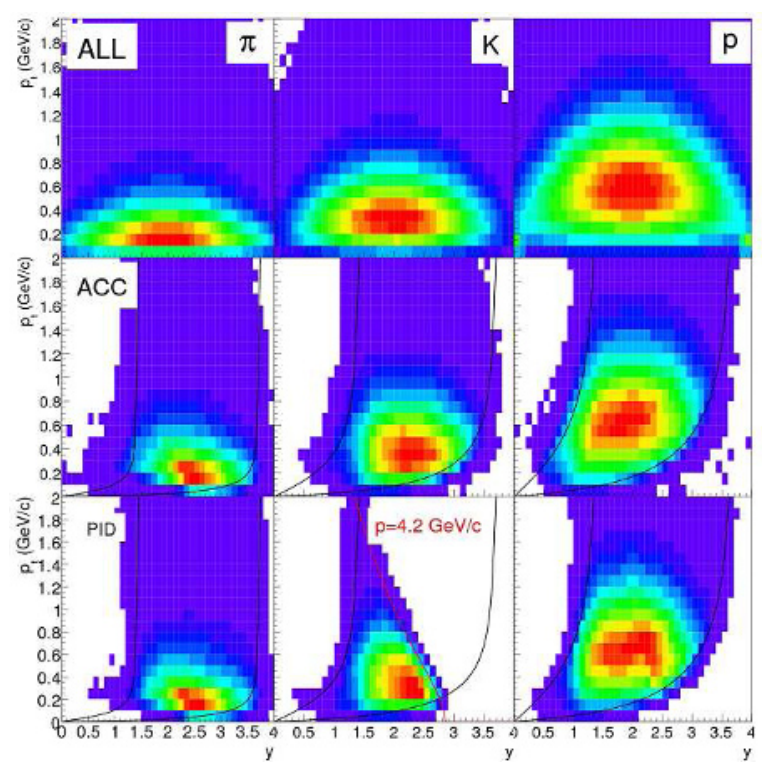

Fig. 6. Phase-space coverage of hadrons, produced in $25 \mathrm{~A} \mathrm{GeV}$ $\mathrm{Au}+\mathrm{Au}$ collisions, in the $\mathrm{CBM}$ detector.

realized with a two-station micro vertex detector in front of the silicon tracker, built from monolithic active pixel sensors [10]. The stations must be ultra-thin. Two MVD stations with a material budget of $0.3 \%$ and $0.5 \%$ radiation lengths have been studied at $5 \mathrm{~cm}$ and $10 \mathrm{~cm}$ downstream of the target. No kaon and proton identification is performed but proton rejection via TOF. The interaction rate is limited to $10^{5}-10^{6}$ per second as imposed by the expected maximum readout speed of the monolithic pixel detectors. With this MVD system the estimate of the open charm yield [11] is, according to the HSD (SHM) models, $16 \mathrm{k}(87 \mathrm{k}) \mathrm{D}^{0}+46 \mathrm{k}(251) \overline{\mathrm{D}}^{0}$ and $26 \mathrm{k}(52 \mathrm{k}) \mathrm{D}^{+}+49 \mathrm{k}$ (98k) $\mathrm{D}^{-}$in $10^{12} 25 \mathrm{~A} \mathrm{GeV} \mathrm{Au+Au} \mathrm{minimum} \mathrm{bias} \mathrm{colli-}$ 

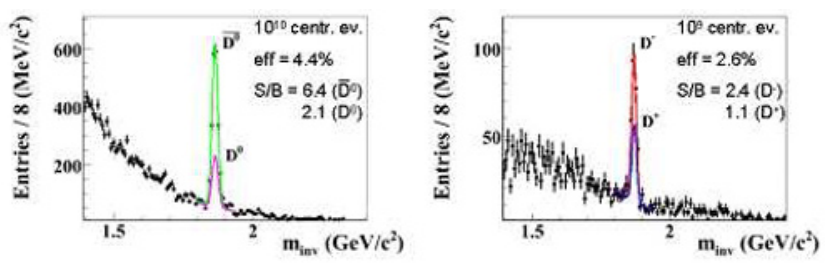

Fig. 7. Invariant mass spectra of $\mathrm{D}$ mesons in central $\mathrm{Au}+\mathrm{Au}$ collisions at $25 \mathrm{~A} \mathrm{GeV}$.

sions. This corresponds to about 2 - 20 weeks of full-time data taking with efficiencies as shown in Fig. 7.

\subsection{Development of detectors, data acquisition system and on-line event selection}

Further developments comprise self-triggering front-end electronics for the STS, RICH and MUCH systems, a readout chip for the TOF system with 25 ps time resolution, a data acquisition system with $500 \mathrm{MB} / \mathrm{s} /$ node throughput, and fast on-line event selection and track reconstruction. For the on-line computing many-core architectures and parallelized reconstruction code are explored [12]. A starting point is the application of commercial high-end graphics cards assembled into a GPU farm. The maximum archiving rate will be $25 \mathrm{kHz}$. High-level trigger strategies are being developed for different physics cases, including:

(a) Open charm: full event reconstruction; limited by MVD readout speed to $10^{5}-10^{6}$ events/s;

(b) $\mathrm{J} / \psi, \omega, \phi \rightarrow \mu^{+} \mu^{-}$: event pre-selection by MUCH (factor $\left.10^{-3}\right)$

(c) $\mathrm{J} / \psi \rightarrow \mathrm{e}^{+} \mathrm{e}^{-}$: trigger based on TRD and STS (minimum bias events).

\subsection{Physics with HADES and pre-CBM at SIS-100}

In the initial phase of FAIR the beams available from the SIS-100 synchrotron allow starting the investigation of nuclear matter in the vicinity of the expected deconfinement phase transition. The currently operating fixed-target experiment HADES at the SIS-18 synchrotron will be moved into the CBM experimental hall and installed in front of a partial configuration of the CBM experiment. The set-up is illustrated in Fig. 8. The combination of the HADES detector, capable of hadron, electron and photon measurement, and the pre-CBM detector with silicon tracking, decay vertex identification, hadron identification and muon detection capabilities, will allow for investigating in separate HADES/CBM data taking runs several observables. These include the excitation function of multi-strange particles (see Fig. 9) and lepton pairs (HADES: electrons, CBM: muons) in heavy-ion collisions, open-charm production at threshold in $\mathrm{p}+\mathrm{C}$ collisions up to $29 \mathrm{GeV}$ (CBM configuration: STS and MVD in dipole magnet, TOF) as well as charmonium production (CBM: STS and reduced muon detector) (see Fig. 10). Cold nuclear matter effects can be studied as reference data for later A+A collisions at SIS-300.

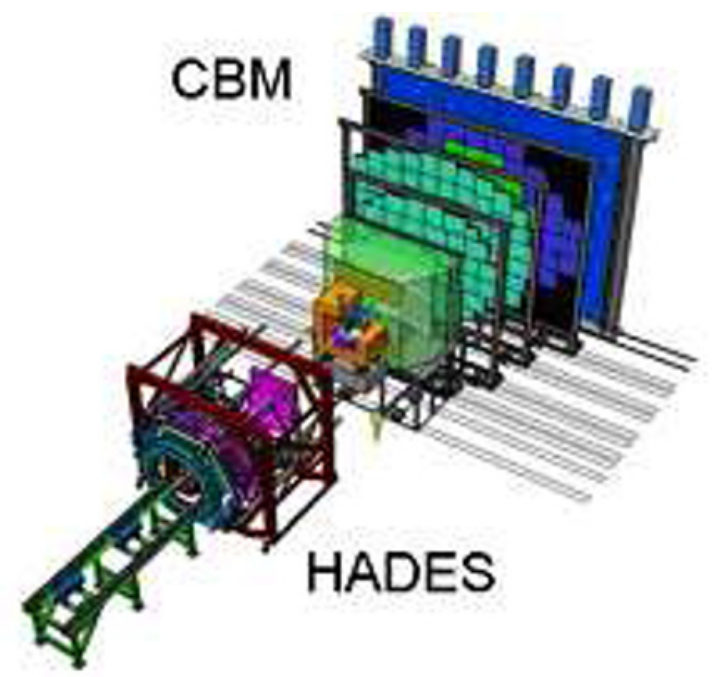

Fig. 8. Illustration of the HADES and CBM detectors in the experimental hall at SIS-100.
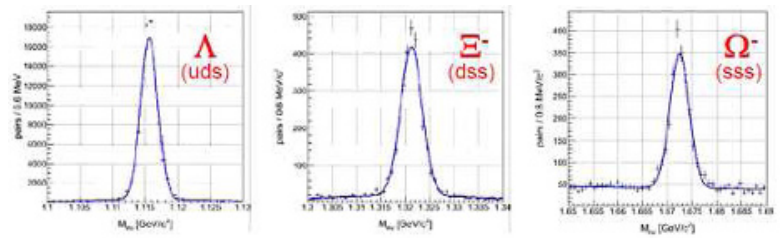

Fig. 9. Invariant mass spectra of hyperons obtained through reconstruction of their decay topology in the CBM silicon tracking system.
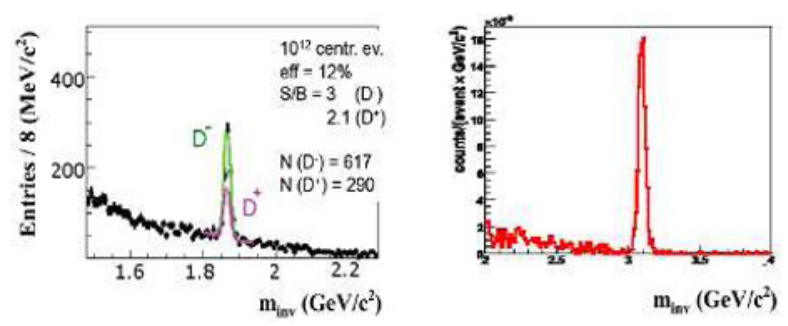

Fig. 10. Charm production in $\mathrm{p}+\mathrm{C}$ collisions at $29 \mathrm{GeV}$ : Open charm from $\mathrm{D}^{ \pm}+\mathrm{X}, \mathrm{D}^{ \pm} \rightarrow \mathrm{K} \pi \pi$ (left) and charmonium from $\mathrm{J} / \psi+\mathrm{X}, \mathrm{J} / \psi \rightarrow \mu^{+} \mu^{-}$(right).

\section{Acknowledgements}

The work reported on has received support from the EU Integrated Infrastructure Initiatives HadronPhysics (I3HP) and HadronPhysics2 (grant No. 227431), the EU INTAS initiative (grant 06-1000012-8729), the Ministry of Science and Technology of China (grant 2008CB817707), the National Natural Science Foundation of China (grants 10620210287, 10610285, 10675072, 10775082, and 10875120 ), the Bundesministerium für Bildung und Forschung, Germany (06DR9059D, 06FY1731, 06HD190i, 06MN229I, 06WU9195I), GSI Helmholtzzentrum für Schwerionenforschung $\mathrm{GmbH}$, the Helmholtz International Center for FAIR (HIC for FAIR), the Hessian LOEWE initiative through HIC for FAIR, the Helmholtz Alliance HA216/ EMMI, the Helmholtz Research School H-QM, the Roma- 
nian NASR/CAPACITATI-Modul III contract nr. 42 and NASR/ PARTENERIATE contract no. 71-144, the Russian Foundation for Basic Research (grant 08-01-00800), and the Federal Agency of Russia for Atomic Energy (Rosatom).

\section{References}

1. C. Höhne, Internat. J. Mod. Phys. E16 (2007) 2419

2. B. Friman, C. Höhne, J. Knoll, S. Leupold, J. Randrup, R. Rapp, P. Senger (Eds.), Lecture Notes in Physics 814 (2010)

3. Nucl. Phys. News $\mathbf{1 6 . 1}$ (2006)

4. https://www.gsi.de/documents/DOC-2009-Nov-1241.pdf

5. Eur. Phys. J. A41 (2009) 243-277

6. CBM Progress Report 2009.

7. J.M. Heuser, PoS VERTEX 2008 (2009) 17

8. C. Höhne, J. Phys. G 35 (2008) 104160

9. D. Gonzalez-Diaz et al., GSI Scientific Report (2006) 11

10. M. Deveaux et al., PoS VERTEX 2008 (2009) 28

11. I. Vassiliev, Proc. NPAE-Kyiv2010, to be published

12. I. Kisel, Nucl. Instr. Meth. Phys. Res. A566 (2008) 8588 